\title{
Comparison of the Electronic Structures of Two Non-cuprate Layered Transition Metal Oxide Superconductors
}

\author{
E. R. Ylvisaker, K.-W. Lee, and W. E. Pickett \\ Department of Physics, University of California, Davis, California, 95616
}

(Dated: December 21, 2018)

\begin{abstract}
Comparison is made of the electronic structure of the little-studied layered transition metal oxide $\mathrm{LiNbO}_{2}$ with that of $\mathrm{Na}_{x} \mathrm{CoO}_{2}$, which has attracted tremendous interest since superconductivity was discovered in its hydrate. Although the active transition metal $d$ states are quite different due to different crystal fields and band filling, both systems show a strong change of electronic structure with changes in the distance between the transition metal ion layer and the oxygen layers. The niobate is unusual in having a large second-neighbor hopping amplitude, and a nearest neighbor hopping amplitude that is sensitive to the $\mathrm{Nb}-\mathrm{O}$ separation. $\mathrm{Li}_{x} \mathrm{NbO}_{2}$ also presents the attractive simplicity of a single band triangular lattice system with variable carrier concentration that is superconducting.
\end{abstract}

\section{MOTIVATION}

Among the various areas of research that were stimulated by the discovery of high temperature superconductors (HTS) nearly two decades ago is that of two-dimensional (2D) (or nearly so) transition metal oxides (TMOs). A second surprise appeared in 2001 with the discovery [1] of $\mathrm{T}_{c}=40$ $\mathrm{K}$ in $\mathrm{MgB}_{2}$, where the physics is entirely different but the 2D character is crucial 2, 3] for the surprisingly high value of critical temperature $T_{c}$. A further stimulus for study of superconductivity in 2D TMOs was provided in 2003 with the discovery of superconductivity [4] in hydrated $\mathrm{Na}_{x} \mathrm{CoO}_{2}$ at 4.5 $\mathrm{K}$. These discoveries suggest a more general look at superconducting 2D TMOs besides the cuprates, to try to identify trends (or perhaps lack of trends).

Being isostructural to the first HTS (La, $\mathrm{Sr})_{2} \mathrm{CuO}_{4}$, the ruthenate $\mathrm{Sr}_{2} \mathrm{RuO}_{4}$ has a special status in this class. Its electronic structure is quite distinct from that of HTS, however, and $\mathrm{T}_{c}$ is only around $1 \mathrm{~K}$. There is now a very large literature on $\mathrm{Sr}_{2} \mathrm{RuO}_{4}$. It is a different and very perplexing superconductor, but we will not pursue it in this paper.

What we focus on here is the little-noticed layered TMO superconductor $\mathrm{Li}_{x} \mathrm{NbO}_{2}$, with brief comparison with the cobaltate system $\mathrm{Na}_{x} \mathrm{CoO}_{2}$. This niobate was discovered [5] in 1990 when the community was absorbed with the new HTS materials, and has not yet attracted the attention that it deserves. While its $\mathrm{T}_{c}=5.5 \mathrm{~K}$ is quite close to that of the hydrated cobaltates $(4.5 \mathrm{~K})$, it is the contrasts that we will focus on. These differences revolve mainly on: $4 d$ versus $3 d$ ion, trigonal versus octahedral coordination by six oxygen neighbors, and single band versus multiband character. We expose one similarity: $z$-displacement of the oxygen layers, which modulates the TM-oxygen distance, has a strong in- fluence on the electronic structure.

\section{LAYERED LITHIUM NIOBATE}

The compound $\mathrm{LiNbO}_{2}$ itself is a band insulator with gap $\sim 2 \mathrm{eV}$. The de-lithiated phase $\mathrm{Li}_{x} \mathrm{NbO}_{2}$ was found by the Berkeley group to be superconducting, [6] with the few reports to date suggesting superconductivity sets in around $x \approx 0.8$ (i.e. when $20 \%$ of the $\mathrm{Li}$ is removed), beyond which $\mathrm{T}_{c}$ does not depend much on the Li content $x$. The structure of $\mathrm{LiNbO}_{2}$ consists of a triangular lattice of both the Li cations and the transition metal (niobium) ions, separated by layers of oxygen atoms, similar to $\mathrm{Na}_{x} \mathrm{CoO}_{2}$ except for the TM coordination. The trigonal prismatic coordination of niobium atoms by oxygen ions provides a big distinction. The trigonal crystal field results in an energetic lowering of the $\mathrm{Nb} \mathrm{d}_{z^{2}}$ states with respect to the other $4 d$ states by about $4 \mathrm{eV}$, leaving the system with only a single band per formula unit to consider. This valence-conduction band is also well separated from the O $2 p$ bands below (see Fig. 1).

Removal of the lithium has the effect of adding holes to the conduction band made up of $\mathrm{Nb} d_{z^{2}}$ states. Superconductivity appears, as it does when holes are introduced into $\mathrm{NaCoO}_{2}$ (followed by hydration), and at a very similar temperature $(5 \mathrm{~K})$, but apparently at quite different carrier concentrations and for very different electronic structures. Since the Li content is variable, this compound becomes a clean representation of a single band triangular lattice system which can be compared rather directly with Hubbard model results. As part of our study of this system, we obtain a tight-binding (TB) representation of the band to allow the subsequent study of possible correlation effects within the Hubbard model. We return to these issues below.

Structure. $\quad \mathrm{LiNbO}_{2}$ takes on a hexagonal 


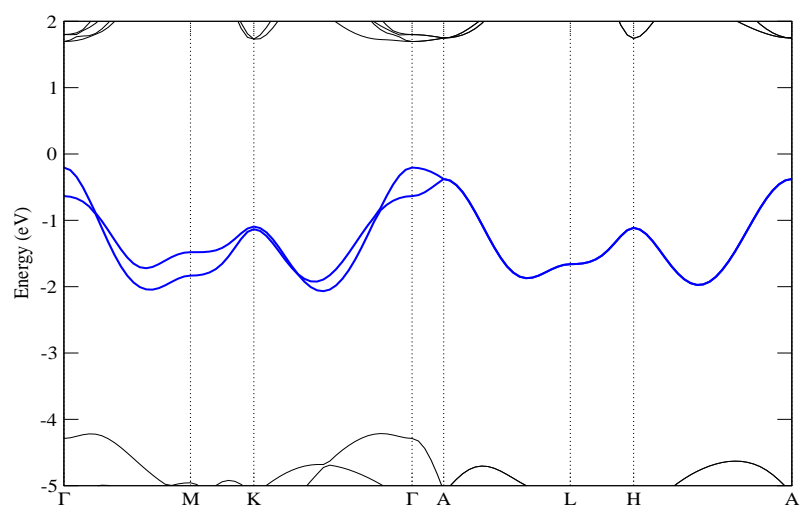

FIG. 1: Band structure for $z_{O}=0.1263$ (one of the experimental values, slightly larger than our optimized value of 0.125), calculated using the code abinit. 13] Symmetry dictates that the two band stick together along the A-L-H-A lines $\left(k_{z} c=\pi\right)$. Non-monotonicity of the bands along the A-L and A-H directions reflects the strong deviation from a nearest-neighbor hopping behavior, as discussed further in the text. The crystal field splitting of the $\mathrm{Nb} 4 d$ bands is $\sim 4 \mathrm{eV}$.

structure [6, 7, 8] ( $a=2.90 \AA, c=10.46 \AA)$ having space group $P 6_{3} / m m c$ (No. 194), with sites $\mathrm{Li}$ $[2 a(0,0,0), \overline{3} m], \mathrm{Nb}\left[2 d\left(\frac{2}{3}, \frac{1}{3}, \frac{1}{4}\right), \overline{6} m 2\right]$, and $\mathrm{O}[4 f$ $\left.\left(\frac{1}{3}, \frac{2}{3}, z_{O}\right), 3 m\right]$. The oxygen internal parameter $z_{O}$ specifies the Nb-O bond length, and due to the stacking type there are two $\mathrm{LiNbO}_{2}$ layers per cell. The distance between $\mathrm{Nb}$ atoms, $a$, is almost identical to bond length $2.86 \AA$ in elemental bcc Nb, so direct $\mathrm{Nb}-\mathrm{Nb}$ overlap should be kept in mind. Experimental values 6 , 7, 8, 9] of the internal parameter range from $0.125-0.129$. Our optimization by energy minimization using the abinit code gives the value $z_{O}=0.125$ (lattice constants held at the experimental values).

Electronic structure and tight-binding representation. The band structure of $\mathrm{LiNbO}_{2}$ pictured in Fig. 1 is similar to that given earlier by Novikov et al. 10] and indicates a $\mathrm{Nb} d_{z^{2}}$ bandwidth of $1.9 \mathrm{eV}$. The $\mathrm{Nb}$ $d_{z^{2}}$-O $2 p$ bands can be fit straightforwardly to a TB model based on orthonormal Wannier functions on the two $\mathrm{Nb}$ atoms per cell (one Nb per layer). A full description of the results will be given elsewhere, but we provide a synopsis here. There are three important features of the TB fit that we emphasize here. First, a good fit requires rather long range hoppings, up to fourth neighbors within the layer and to three neighbors in the layers above and below. Second, with oxygen ions at their equilibrium position, the second neighbor (in-plane) hopping amplitude $t_{2} \approx$ $100 \mathrm{meV}$ is much larger than the nearest neighbor hopping $t_{1} \approx 60 \mathrm{meV}$. The smaller value of $t_{1}$ may reflect interference between direct $\mathrm{Nb}-\mathrm{Nb}$ interaction and the standard O-mediated $\mathrm{Nb}-\mathrm{O}-\mathrm{Nb}$ processes.
TABLE I: Born effective charges for $\mathrm{LiNbO}_{2}$, together with a comparison with $\mathrm{NaCoO}_{2}$ calculated by $\mathrm{Li}$ et al. 15] The angular average $Z_{a v}^{*}$ is also displayed. Note the unexpected deviations from the formal values $Z^{0}$ of the effective charges for $\mathrm{Li}$ and $\mathrm{Nb}$ in the $z$-direction (larger for $\mathrm{Li}$, smaller for $\mathrm{Nb}$ ). For $\mathrm{O}$ in $\mathrm{LiNbO}_{2}$, the effective charges are nearly isotropic. Overall, the anisotropies are rather similar in $\mathrm{NaCoO}_{2}$, but somewhat more pronounced.

\begin{tabular}{lccccccc}
\hline \hline & \multicolumn{3}{c}{$\mathrm{LiNbO}_{2}$} & & \multicolumn{3}{c}{$\mathrm{NaCoO}_{2}$} \\
\cline { 2 - 4 } \cline { 6 - 8 } & $\mathrm{Li}$ & $\mathrm{Nb}$ & $\mathrm{O}$ & & $\mathrm{Na}$ & $\mathrm{Co}$ & $\mathrm{O}$ \\
\hline $\mathbf{Z}_{x x}^{*}$ & 1.10 & 2.26 & -1.68 & & 0.87 & 2.49 & -1.68 \\
$\mathbf{Z}_{z z}^{*}$ & 1.69 & 1.31 & -1.50 & & 1.37 & 0.87 & -1.12 \\
\hline $\mathbf{Z}_{a v}^{*}$ & 1.30 & 1.94 & -1.62 & & 1.04 & 1.95 & -1.49 \\
\hline $\mathbf{Z}^{0}$ & +1 & +3 & -2 & & +1 & +3 & -2 \\
\hline \hline
\end{tabular}

The same trend has been observed for $2 \mathrm{H}-\mathrm{TaS}_{2}, 11$ where the small value of $t_{1}$ was traced to phase cancellation in the hopping integral when Wannier functions are on nearest neighbors. This $t_{2}>t_{1}$ feature may have important implications for the microscopic understanding of the properties of $\mathrm{Li}_{x} \mathrm{NbO}_{2}$, since if $t_{2}$ were the only nonzero hopping, the system decomposes into three decoupled triangular lattices with lattice constant $\sqrt{3} a$; $t_{1}$ then becomes the "perturbation" that couples the three sublattices, breaks symmetry and removes degeneracy. Thirdly, the nearest neighbor hopping $t_{1}$ is very strongly modulated by oxygen displacement. We find that $t_{1}$ increases strongly as the O layers "squash" against the Nb layers, as in the $A_{g}$ Raman mode. This modulation may provide the largest contribution to electron-phonon coupling in this compound.

Effective charges. We have evaluated the Born effective charge tensor as described by Gonze and Lee 12] using the abinit code. 13] Given in Table [ are the two distinct elements of the effective charge tensor for each atom type, calculated in the relaxed atomic structure, together with the formal charges. $Z_{x x}^{*}(\mathrm{Li})\left(Z_{y y}^{*}=Z_{x x}^{*}\right)$ is close to the formal charge of $\mathrm{Li}$ indicating primarily ionic type bonding for motion in the $x-y$ plane, consistent with its propensity for de-intercalation. The charge tensor for Li shows similar anisotropy to that of LiBC [14], which is similar structurally and electronically (if some $\mathrm{Li}$ is deintercalated) to the $40 \mathrm{~K}$ superconductor $\mathrm{MgB}_{2}$. In $\operatorname{LiBC} Z_{x x}^{*}(\mathrm{Li})=0.81, Z_{z z}^{*}(\mathrm{Li})=1.46$, and it was concluded that $\mathrm{Li}$ is involved in electronic coupling (not only ionic, but covalent) between consecutive B-C layers. Similar Li involvement might be expected in $\mathrm{LiNbO}_{2}$, and indeed the band structure shows clear effects of interlayer coupling. The difference from the formal charges for the $\mathrm{Nb}$ ions (formally $\mathrm{Nb}^{3+}$, $\mathrm{O}^{2-}$ ) indicate substantial covalent character to the 


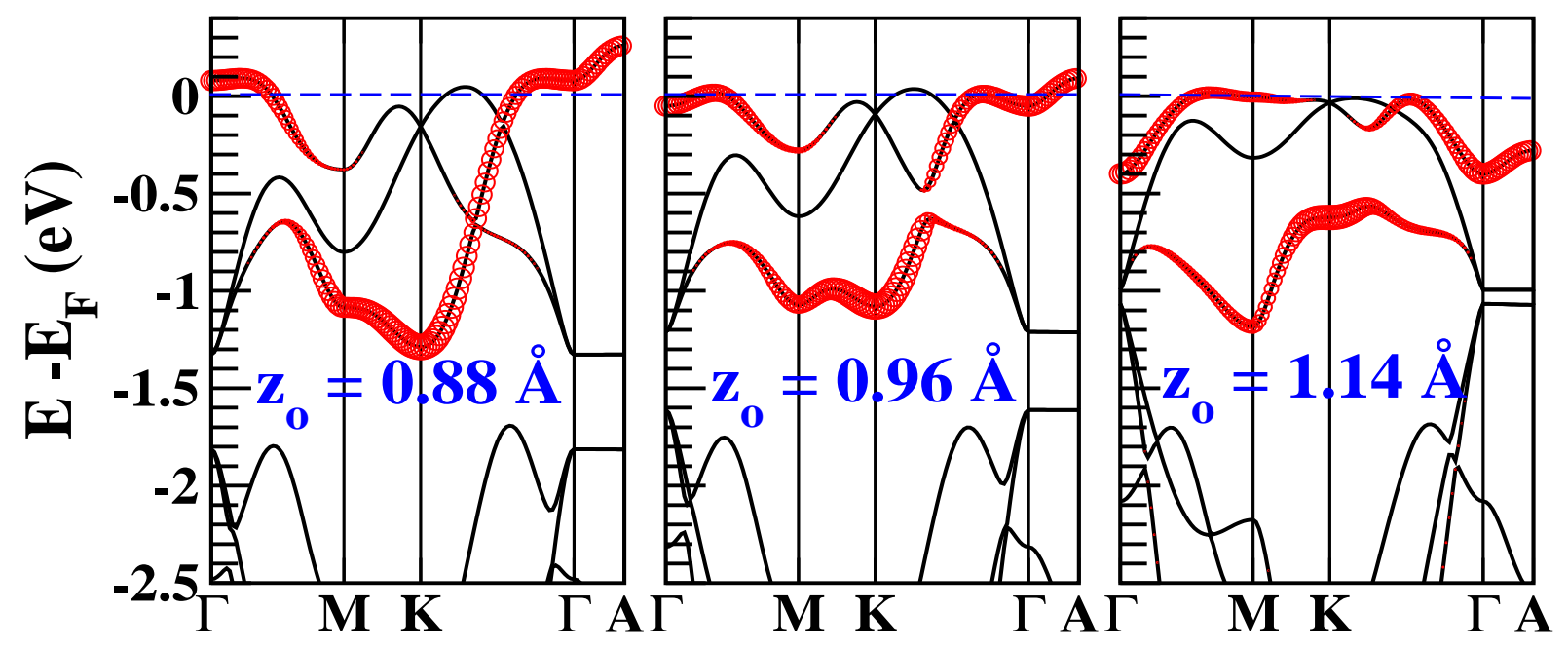

FIG. 2: The $t_{2 g}$ bands (which lie above $-1.5 \mathrm{eV}$ in these figures) of paramagnetic $\mathrm{Na}_{0.5} \mathrm{CoO}_{2}$ for three values of the $\mathrm{O}$ height $z_{O}$. The value $1.14 \AA$ corresponds to symmetric $\mathrm{CoO}_{6}$ octahedra. The thickened line emphasizes the $a_{g}$ character. The changes in the $t_{2 g}$ bands are discussed in the text. Note also the changes in the O $2 p$ bands just below the $t_{2 g}$ bands.

bonding, which appears to be especially strong for $z$ displacement of the $\mathrm{Nb}$ ion.

The Born effective charges have been reported 15$]$ for $\mathrm{NaCoO}_{2}$, and since we investigate $\mathrm{O}$ squashing in this compound in the next section, we have included the $\mathrm{NaCoO}_{2}$ effective charges in Table \for comparison. Indeed there are several similarities, as noted in the table caption.

\section{LAYERED SODIUM COBALTATES}

There is already a substantial literature on the electronic structure of the $\mathrm{Na}_{x} \mathrm{CoO}_{2}$ system. Briefly: the $t_{2 g}$ bands are broken in symmetry by the layered structure and by the squashing of the $\mathrm{CoO}_{2}$ layers away from ideal cubic coordination by the six $\mathrm{O}$ ions. The bands are doped with $1-x$ holes, with all the evidence indicating the holes go, at least initially, into $a_{g}$ states rather than $e_{g}^{\prime}$ states. Using the observed structure, it is found that this results from the somewhat larger $a_{g}$ bandwidth, because the band centers remain indistinguishable.

We address here the effect of the height of the $\mathrm{O}$ ions above/below the Co layer. In the calculations, the full-potential nonorthogonal local-orbital minimum-basis scheme (FPLO) was used. 16] For specific doping levels and treatments of the $\mathrm{Na}$ ions, the height has been optimized by a number of groups [17, 18, 19, 20, 21], revealing that there is some sensitivity of the $\mathrm{O}$ position to the environment. To clarify the question of the effect of squashing without reference to a specific doping level, we

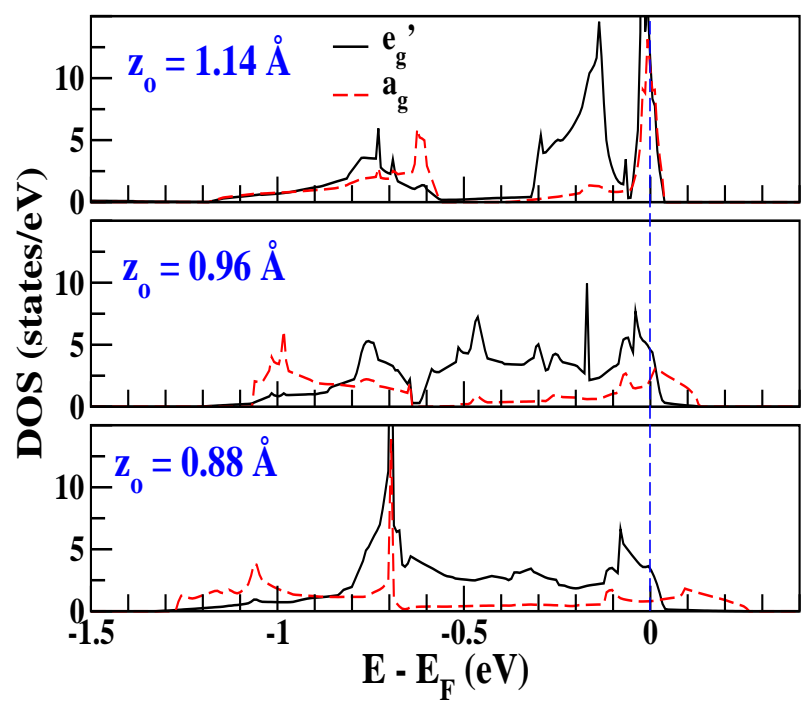

FIG. 3: The $a_{g}$ and $e_{g}^{\prime}$ densities of states for the oxygen heights, and bands, shown in Fig. 2. Note that the band widths are identical for the symmetric octahedra $\left(z_{O}=1.14 \AA\right)$ but the two-dimensional dispersion already results in a strongly differing DOS in the upper regions (where, within a rigid band picture, the doped holes reside).

display in Fig. 2 the $t_{2 g}$ bands for $\mathrm{O}$ height (from the Co layer) of $1.14 \AA$ (corresponding to undistorted $\mathrm{CoO}_{6}$ octahedra), $0.96 \AA$ (typical value for intermediate values of $x$ ), and $0.88 \AA$ (the smallest value reported). For orientation, we note that Johannes 
et al., 18] using the virtual crystal approximation for the Na concentrations $x=0.3,0.5$, and 0.7 , obtained the heights $0.88,0.90,0.93 \AA$ respectively. The corresponding projected densities of states are shown in Fig. 3. For these calculations we used $x=0.5$, treated within the virtual crystal model. To avoid unphysical O-O interactions across the layers as the O layer position was varied, the $c$ axis was artificially increased by $20 \%$ for these calculations.

Simple crystal field arguments would suggest: (1) for the cubic octahedron $z_{O}=1.14 \AA$, the $a_{g}$ and $e_{g}^{\prime}$ DOS should be the same, and (2) as the O ions are squashed down, the $e_{g}^{\prime}$ states should rise relative to the $a_{g}$ states. The first expectation is severely violated in the region just below $E_{F}$ due to the dispersion being only two-dimensional (presuming crystal fields from ions beyond nearest $\mathrm{O}$ ions are negligible). In addition, the effects of squashing are much more complex than suggested by the crystal field model. There is minor change in the mean energies of the $a_{g}$ and $e_{g}^{\prime}$ states (they remain essentially equal, see Fig. 3), the main change is an increase in the $a_{g}$ bandwidth compared to that of the $e_{g}^{\prime}$ states upon squashing. For $z_{O}=1.14 \AA$, doped holes initially would go equally into each band. At the highly squashed end, $\sim 0.4$ holes/per Co can go into the $a_{g}$ band before encountering the $e_{g}^{\prime}$ states. We emphasize that this is a model, constrained result; selfconsistency and geometrical relaxation will change the details. There is also the question of decreasing interaction with the $\mathrm{O} 2 p$ states upon squashing. This change, which is of course also included in the changes shown in Figs. 2 and 3, may affect the $a_{g}$ and $e_{g}^{\prime}$ states differently.

The changes in the band structure, Fig. 2, are more instructive. At $\Gamma$, the $a_{g}$ state is almost 0.5 $\mathrm{eV}$ below its maximum for the cubic octahedron $z_{O}=1.14 \AA$, the maxima occurring midway along both $\Gamma-\mathrm{M}$ and $\Gamma-\mathrm{K}$ lines. The additional struc- ture, and the associated decrease in bandwidth reflects longer range hopping, and most likely a strong change in the ratio $t_{2} / t_{1}$, analogous to the changes in $\mathrm{LiNbO}_{2}$ but with additional complications due to the presence of the $e_{g}^{\prime}$ bands. The shift with squashing motion in the $e_{g}^{\prime}$ bands is noticeable not only at $\Gamma$, where the state increases in energy, but also in the degeneracy at the $\mathrm{K}$ point, which rises to the top of the $t_{2 g}$ bands for the symmetric $\mathrm{CoO}_{6}$ octahedron.

\section{SUMMARY}

In this paper we have briefly compared and contrasted the electronic structure of the little-studied layered TMO $\mathrm{LiNbO}_{2}$ to that of $\mathrm{Na}_{x} \mathrm{CoO}_{2}$, which has attracted tremendous since superconductivity was discovered in its hydrate. Although the active states are quite different, both systems show a strong change of electronic structure with changes in the TM-oxygen distance. The niobate is unusual in having a large second-neighbor hopping amplitude, and it also presents the attractive simplicity of a single active band on a triangular lattice. One of the primary questions to address is whether electronic correlations are important in the delithiated system, and whether the origin of superconductivity is of electronic or lattice origin.

\section{ACKNOWLEDGMENTS}

We acknowledge stimulating comments from D. Khomskii and R. J. Cava on the effect of oxygen "squashing" in the $\mathrm{Na}_{x} \mathrm{CoO}_{2}$ system, and clarification from M. D. Johannes on calculations relating to this question. This work was supported by National Science Foundation Grant DMR-0421810.
[1] J. Nagamitsu, N. Nakagawa, T. Muranaka, Y. Zenitani, and J. Akimitsu, Nature (London) 410, 63 (2001).

[2] I. I. Mazin and V. P. Antropov, Physica C 385, 49 (2003).

[3] W. E. Pickett, Brazilian J. Phys. 33, 695 (2003).

[4] K. Takada, Y. Sasago, E. Takayama-Muromachi, F. Izumi, R. A. Dilanian, and T. Sasaki, Nature (London) 422, 53 (2003).

[5] M. J. Geselbract, T. J. Richardson, and A. M. Stacy, Nature 345, 324 (1990).

[6] M. J. Geselbracht, A. M. Stacy, A. R. Garcia, B. G. Slibernagel, and G. H. Kwei, J. Phys. Chem 97, 7102 (1993).
[7] E. G. Moshopoulou, P. Bordet, and J. J. Capponi, Phys. Rev. B 59, 14 (1999).

[8] G. Meyer and R. Hoppe, Angew. Chem. (Intl. Ed.) 13, 11 (1974).

[9] A. P. Tyutyunnik, V. G. Zubkov, D. G. Kellerman, V. A. Pereliaev, and A. E. Kar'kin, Eur. J. Solid State Inorg. Chem. 33, 53 (1996).

[10] D. L. Novikov, V. A. Gubanov, V. G. Zubkov, and A. J. Freeman, Phys. Rev. B 49, 15830 (1994).

[11] R. L. Barnett, A. Polkovnikov, E, Demler, W.-G. Yin, and $\mathrm{W} . \mathrm{Ku}$, cond-mat/0508590

[12] X. Gonze and C. Lee, Phys. Rev. B 55, 10355 (1997).

[13] X. Gonze, J.-M. Beuken, R. Caracas, F. Detraux, M. 
Fuchs, G.-M. Rignanese, L. Sindic, M. Verstraete, G. Zerah, F. Jollet, M. Torrent, A. Roy, M. Mikami, Ph. Ghosez, J.-Y. Raty, and D.C. Allan, Comput. Mater. Sci. 25, 478 (2002); The ABINIT code is a common project of the Université Catholique de Louvain, Corning Incorporated, and other contributors (URL http://www.abinit.org).

[14] K.-W. Lee and W. E. Pickett, Phys. Rev. B 68, 085308 (2003).

[15] Z. Li, J. Yang, J. G. Hou, and Q. Zhu, Phys. Rev. B 70, 144518 (2004).

[16] K. Koepernik and H. Eschrig, Phys. Rev. B 59, 1743
(1999).

[17] P. Zhang, W. Luo, V. H. Crespi, M. L. Cohen, and S. G. Louie, Phys. Rev. B 70, 085108 (2004).

[18] M. D. Johannes, D. A. Papaconstantopoulos, D. J. Singh, and M. J. Mehl, Europhys. Lett. 68433 (2004).

[19] J. Ni and G. Zhang, Phys. Rev. B 69, 214503 (2004).

[20] Z. Li, J. Yang, J. G. Hou, and Q. Zhu, Phys. Rev. B 71, 024502 (2005).

[21] K.-W. Lee and W. E. Pickett, Phys. Rev. B 72, 115110 (2005). 